\title{
NEW POSSIBILITIES FOR INVESTIGATION OF THE TECHNOLOGICAL TEXTURE BASED ON MEASUREMENT OF ELECTRIC PARAMETERS: THEORETICAL ANALYSIS AND EXPERIMENTAL VERIFICATION
}

\author{
Tomáš Kozík ${ }^{*}$ - Stanislav Minárik ${ }^{* *}, * * *$
}

\begin{abstract}
Texture is preferred orientation of crystallites in some polycrystalline materials. Different methods are applied to characterize the orientation patterns and determine the orientation distribution. Most of these methods rely on diffraction.

This paper introduces the principle of a method used for characterization of ceramics texture based on anisotropy of electrical properties of crystallites in ceramics. The mathematical framework of this method is presented in theoretical part of our work. In experimental section we demonstrate how the theoretical result could be used to evaluate technology texture of ceramic material intended for the production of electronic insulators.
\end{abstract}

K e y w o r d s: ceramics texture, scalar potential, Laplace equation, Bessel function, Bessel differential equation

\section{INTRODUCTION}

The distribution of crystallographic orientations of a polycrystalline dielectric (texture) has a considerable impact on application properties of the material. The texture arising in the manufacture process of cylindrical insulators (technological texture) has a detrimental effect on insulators functionality.

Geometric shape of the particles of plastic component of ceramic mixture (kaolins and clays), arrangement and a shape of the end of the extruder, size of the blank (especially the specific dimension of the section of the blank) and rheological properties of ceramic mixture for producing bulk ceramic bodies (insulators) determine the spatial arrangement and orientation of ceramic particles of particular components of the ceramic mixture in the blank. The created arrangement of the particles of particular components in the blank - the technological texture - significantly affects the technological properties of the blank in individual technological operations (cutting, drying, glazing, firing) and also affects the final properties of the product, hence the efficiency of the entire production process.

One of the unfavorable impacts of technological texture on production quality after firing are various cracks on the surface and in the volume of the insulator, Fig.1.

Technological texture negatively affects the mechanical strength of the insulator itself after firing, which is a qualitative parameter of the electrical product - insulator. Knowledge of the topography of technological texture is a prerequisite for achieving high efficiency and of production quality of $\mathrm{VHV}$ insulators.

It is therefore advisable to check the technological texture of dielectric blank directly in the production process of insulators.
In general, texture can be investigated by various quantitative techniques (X-ray diffraction, ESBD, SEM, etc) and qualitative analysis (polarized microscope, Laue photography, pole figure technique, etc). All of the laboratory methods mentioned above are not applicable when we check the technological texture of serial products because they require quite complicated technical equipment and procedures. In our work we introduce the principle of a simple method for the evaluation of axial technological texture in dielectric material. The main idea is based on the investigation of electrostatic field axial distribution in thin cylindrical samples made from dielectric material.

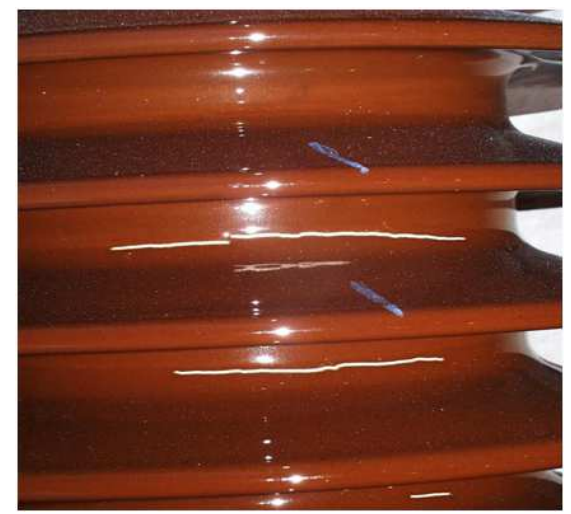

Fig. 1. Crack in the body of insulator

We calculate the electrostatic field distribution in thin homogeneous cylindrical dielectric sample inserted between electrodes of the capacitor that has circular electrode with unequal diameter. Especially attention is paid to axially symmetric field dissipation in the area behind the edge of the circular electrode with smaller radius. Generally, the typical case of Sturm-Liouville boundary

\footnotetext{
* Constantine the Philosopher University in Nitra, Dražovská cesta 4, 94974 Nitra, Slovakia, kozik@slovanet.sk ** Research Institute of Advanced Technologies, Faculty of Materials Science and Technology STU Bratislava, Paulínska 16, CAMBO, 917 24 Trnava, Slovakia, *** Institute of Ion-Beam Physics and Materials Research, Helmholtz-Zentrum Dresden-Rossendorf, Bautzner Landstrasse 400, 01328 Dresden, Germany
} 
value problem called Bessel differential equation arises in calculation of the scalar potential with axial symmetry. After finding the particular solution of this equation using the Bessel functions the difference of potential of the electrostatic fields in material is expressed. Consequently we consider a limit case of extremely slim capacitor and investigate the decrease of the difference of the potential in the electrostatic field in the area behind the edge of smaller electrode by means of the asymptotic Bessel functions.

Further we propose how to use the result of theoretical analysis for the checking of technological texture in practice. Experimentally measured axial distribution of electrostatic potential differs from the theoretical values in some measurement points. These differences are due to local variations of dielectric properties of material and they allow to evaluate the axial technology texture.

\section{THEORY}

We consider a cylindrical dielectric sample with radius $R_{1}$ and very small thickness $\left(h<<R_{2}\right)$ inserted between two parallel coaxial metal electrodes with radii $R_{1}>R_{2}$. Voltage $U$ is applied to electrodes, Fig. 2.

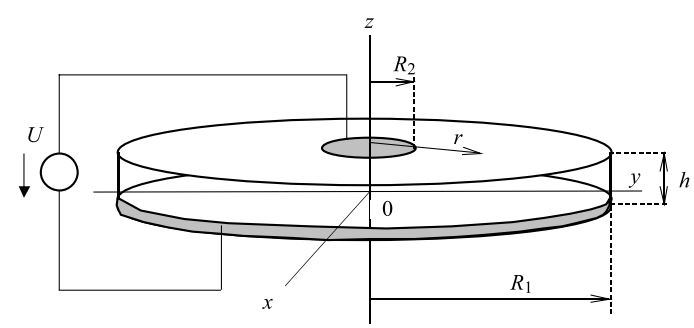

Fig. 2. Experimantal layout

Potential of electrode with radius $R_{1}$ is $\varphi_{1}$ and the potential of electrode with radius $R_{2}$ is $\varphi_{2}$. We are interested in electrostatic field potential $\varphi$ dependence on distance $r$ from the axis of both plates measured on the sample surface in area $R_{2} \leq r \leq R_{1}$ for $z=h$, according to Fig. 2.

\subsection{The solution of Laplace equation for axially symmetric electrostatic field}

It is possible to consider Laplace equation $[1,2]$ for charge-free electrostatic field in investigation of scalar potential distribution in thin cylindrical dielectric media. The mentioned equation can be written in cylindrical coordinates

$$
\frac{\partial^{2} \varphi}{\partial r^{2}}+\frac{1}{r} \frac{\partial \varphi}{\partial r}+\frac{1}{r^{2}} \frac{\partial^{2} \varphi}{\partial \theta^{2}}+\frac{\partial^{2} \varphi}{\partial z^{2}}=0
$$

If we assume axial symmetry of homogeneous dielectric media $\partial^{2} \varphi / \partial \theta^{2}=0$ and we expect a separable solution

$$
\varphi(r, z)=\Phi(r) Y(z)
$$

It results from the Laplace equation that components $\operatorname{Phi}(r)$ and $Y(z)$ must obey next equations

$$
\begin{gathered}
\frac{\partial^{2} \Phi(r)}{\partial r^{2}}+\frac{1}{r} \frac{\partial \Phi(r)}{\partial r}=\lambda \Phi(r) \\
\frac{\partial^{2} Y(z)}{\partial z^{2}}=-\lambda Y(z)
\end{gathered}
$$

where $\lambda$ is a constant that can be positive, negative or equal to zero.

a) If $\lambda=0$ we can easily find the solution of (1) in the following form

$$
\varphi_{0}(r, z)=K z \ln r+L \ln r+M z+N
$$

where $K, L, M$ and $N$ are constants of integration depending on the boundary conditions.

b) In case if $\lambda>0$ the solution of (4) can be found in the form

$$
\begin{aligned}
& \qquad Y_{p}(z)=Y_{1} \sin (k z+\alpha) \\
& \text { where } \lambda=k^{2}, \quad \text { and } \quad Y_{1}-\text { is a constant }
\end{aligned}
$$

Equation (4) can be transformed to a special case of modified Bessel differential equation

$$
x^{2} \frac{\partial^{2} \Phi}{\partial x^{2}}+x \frac{\partial \Phi}{\partial x}-x^{2} \Phi=0
$$

where $x=k r$. Next, the solution of (3) can be determined

$$
\Phi_{p}(r)=A_{2} I_{0}(k r)+B_{2} K_{0}(k r)
$$

where $A_{2}$ and $B_{2}$ are constants of integration and $I_{0}(k r)$ and $K_{0}(k r)$ are modified Bessel functions of zero order $[3,4]$

$$
\begin{gathered}
I_{0}(x)=\sum_{m=0}^{\infty} \frac{1}{(m !)^{2}}\left(\frac{x}{2}\right)^{2 m} \\
K_{0}(x)=-I_{0}(x)\left\{\eta+\ln \left(\frac{x}{2}\right)\right\}+ \\
\frac{2}{1} I_{2}(x)+\frac{2}{2} I_{4}(x)+\frac{2}{3} I_{6}(x)+\ldots
\end{gathered}
$$

c) In case of $\lambda<0$ the solution of (4)is

$$
Y(x)=A_{3} e^{k z}+B_{3} e^{-k z}
$$

where $\lambda=-k^{2}, A_{3}$ and $B_{3}$ are constants of integration. Equation (3) can be transformed to

$$
x^{2} \frac{\partial^{2} \Phi}{\partial x^{2}}+x \frac{\partial \Phi}{\partial x}+x^{2} \Phi=0
$$

with $x=k r$. Equation (10) is also a special case of Bessel differential equation [4,5] and the solution of (4) can be written as

$$
\Phi_{p}^{(2)}(x)=K_{2} I_{0}(k r)+M_{2} Y_{0}(k r)
$$


where where $K_{2}$ and $M_{2}$ are constants of integration and

$$
\begin{gathered}
J_{0}(x)=\sum_{m=0}^{\infty} \frac{(-1)^{m}}{(m !)^{2}}\left(\frac{x}{2}\right)^{2 m} \\
Y_{0}(x)=\frac{2}{\pi}\left[J_{0}(x)\left\{C+\ln \left(\frac{x}{2}\right)\right\}\right]+\frac{2}{1} J_{2}(x) \\
\left.-\frac{2}{2} J_{4}(x)+\frac{2}{3} J_{6}(x)+\ldots\right]
\end{gathered}
$$

are the Bessel functions of zero order. Subsequently, the general solution of (1) can be found

$$
\begin{aligned}
\varphi^{(1)}(r, z) & =Y^{0}\left[A_{2} I_{0}(k r) B_{2} K_{0}(k r)\right] \sin (k z+\alpha)+ \\
& +K z \ln r+L \ln r+M z+N, \quad \text { if } \quad \lambda>0 \\
\varphi^{2}(r, z)= & {\left[K_{2} J_{0}(k r)+M_{2} Y_{0}(k r)\right]\left(A_{3} e^{k z}+B_{3} e^{-k z}\right) } \\
+ & K z \ln r+L \ln r+M z+N, \quad \text { if } \quad \lambda<0
\end{aligned}
$$

\subsection{Application of boundary conditions}

Let the distribution of scalar potential be determined by a function $\phi^{(I)}(r, z)$ in area $0 \leqslant r \leqslant R_{2}, z \leqslant 0 \leqslant h$ where this must obey the following conditions

$$
\phi^{(I)}(r, 0)-\varphi_{1}, \quad \phi^{(I)}(r, h)-\varphi_{2}
$$

For this reason, function $\phi^{(I)}(r, z)$ must be written as (12) and the following conditions must be satisfied $\sin (k h+\alpha)=0$. This means $k h+\alpha=n \pi$, where $n, m=0, \pm 1, \pm 2, \ldots)$. There is no reason for the periodicity of solution $\phi^{(I)}(r, z)$. Therefore, it is possible to take into account $\alpha=0, k=\pi / h$. Boundary conditions (14) can be satisfied only in case if $K=0$ and $L=0$. For that reason $\phi^{(I)}(r, z)$ must be written in the following form

$$
\begin{aligned}
\varphi^{(I)}(r, z) & =\left[A_{2} I_{0}\left(\frac{\pi}{h} r\right)+B_{2} K_{0}\left(\frac{\pi}{h} r\right)\right] \sin \left(\frac{\pi}{h} z\right)+ \\
& +M z+N
\end{aligned}
$$

Integration constants can be determined by substituting (15) to (14)

$$
N=\varphi_{1}, \quad M=\frac{\varphi_{2}-\varphi_{1}}{h}=\frac{U}{h}
$$

and the distribution of scalar potential in this area takes the form

$$
\begin{aligned}
\varphi^{(I)}(r, z) & =\left[A_{2} I_{0}\left(\frac{\pi}{h} r\right)+B_{2} K_{0}\left(\frac{\pi}{h} r\right)\right] \sin \left(\frac{\pi}{h} z\right)+ \\
& +\frac{U}{h} z+\varphi_{1} .
\end{aligned}
$$

Let the distribution of scalar potential be determined by function $\varphi^{(I I)}(r, z)$ in area $R_{2} \leqslant r \leqslant R_{1}, z \leqslant 0 \leqslant$ $h$ (area II). Function $\varphi^{(I I)}(r, z)$ must obey the following conditions

$$
\phi^{(I I)}(r, 0)-\varphi_{1}, \quad \phi^{(I I)}\left(R_{2}, z\right)=\phi^{(I)}\left(R_{2}, z\right)
$$

Therefore, function $\varphi^{(I I)}(r, z)$ must be also written in the form (12). The boundary conditions (18) can be satisfied only in case if $L=0$ and $N=\varphi_{1}$. If we consider the following substitution

$$
M=-K \ln r_{0}
$$

where $r_{0}$ is constant, the distribution of scalar potential can be written as follows

$$
\begin{aligned}
\varphi^{(I I)}(r, z)= & Y^{0}\left[A_{2} I_{0}\left(\frac{\pi}{h} r\right)+B_{2} K_{0}\left(\frac{\pi}{h} r\right)\right] \sin \left(\frac{\pi}{h} z\right) \\
& +K z \ln \left(\frac{r}{r_{0}}\right)+\varphi_{1}
\end{aligned}
$$

We assume that $r>>h$, then $\pi r / h>>1$ and arguments of the Bessel functions are very large. It holds for very large values of arguments in this case $[4,5]$

$$
\begin{array}{r}
I_{0}\left(\frac{\pi}{h} r\right) \rightarrow \frac{1}{\pi} \sqrt{\frac{h}{2 r}} e^{\frac{\pi}{h} r} \\
K_{0}\left(\frac{\pi}{h} r\right) \rightarrow \sqrt{\frac{h}{2 r}} e^{-\frac{\pi}{h} r} \rightarrow 0
\end{array}
$$

In addition, function $\varphi^{(I I)}(r, z)$ must be decreasing and $A_{2}=0$ for that reason. Consequently, the distribution of scalar potential in thin dielectric media inserted between electrodes is determined by the following functions

$$
\begin{aligned}
\phi^{(I)}(r, z) & =\frac{U}{h} z+\varphi_{1} \\
\varphi^{(I I)}(r, z) & =K z \ln \left(\frac{r}{r_{0}}\right)+\varphi_{1}
\end{aligned}
$$

After substituting (21) and (22) to second equation (18) we obtain

$$
K=\frac{U}{h \ln \left(\frac{R_{2}}{r_{0}}\right)}
$$

We can find constant $r_{0}$ based on the fact that total electric charge $Q$ accumulated on both electrodes is the same. In the case of thin homogeneous sample we assume

$$
\begin{gathered}
Q=\varepsilon \int_{(S)} \sigma d S=\varepsilon \int_{0}^{R_{2}} \sigma^{(I)}(r) 2 \pi r d r= \\
\varepsilon \int_{0}^{R_{2}} \sigma^{(I)}(r) 2 \pi r d r+\varepsilon \int_{R_{2}}^{R_{1}} \sigma^{(I I)}(r) 2 \pi r d r
\end{gathered}
$$

where $\varepsilon$ is electric permittivity of the material $(\varepsilon=$ const in homogeneous case) and $\sigma^{(I)}, \sigma^{(I I)}$, are the charge 
densities on electrodes surface (in area I and area II). The following applies

$$
\begin{array}{ll}
\sigma^{(I)}=\varepsilon E_{z}^{(I)}(r, 0) & \text { for } r \leq R_{2} \\
\sigma^{(I I)}=\varepsilon E_{z}^{(I I)}(r, 0) & \text { for } R_{2} \leq r \leq R_{1}
\end{array}
$$

where $E_{z}^{(I)}, E_{z}^{(I I)}$ are $z$-components of electrostatic intensity vector in areas I and II respectively. We consider

$$
\begin{aligned}
& E_{z}^{(I)}=-\frac{\partial \varphi^{(I)}}{\partial z}=-\frac{U}{h} \\
& E_{z}^{(I I)}=-\frac{\partial \varphi^{(I I)}}{\partial z}=-K_{0} \ln \left(\frac{r}{r_{0}}\right) .
\end{aligned}
$$

If we further consider (24) we obtain

$$
\int_{R_{1}}^{R_{2}} r \ln \left(\frac{r}{r_{0}}\right) \mathrm{d} r=0
$$

After integrating (27) we can determine $r_{0}$

$$
r_{0}=\frac{R_{2}}{\sqrt{e}}\left(\frac{R_{2}}{R_{1}}\right)^{\frac{R_{1}^{2}}{R_{2}^{2}-R_{1}^{2}}}
$$

and $K$ by substituting (28) to (23), consequently

$$
K=\frac{\frac{U}{h}}{\left(\frac{R_{1}^{2}}{R_{2}^{2}-R_{1}^{2}} \ln \frac{R_{1}}{R_{2}}+\frac{1}{2}\right)}
$$

We can find the distribution of scalar potential in the area II on the surface of dielectric media $(z=h)$ by substituting (29) (28) to (22). Next, the voltage measured on the surface of thin sample can be evaluated by

$$
\begin{gathered}
U_{v}(r, h)=\varphi^{(I I)}(r, h)-\varphi_{1} \approx\left(1-\eta \ln \frac{r}{R_{2}}\right) U \\
\text { where: } \quad \eta=\frac{1}{\frac{R_{1}^{2}}{R_{1}^{2}-R_{2}^{2}} \ln \frac{R_{1}}{R_{2}}-\frac{1}{2}}
\end{gathered}
$$

\section{EXPERIMENT}

Determination of the electrostatic potential distribution on the surface of the sample was carried out according to wiring diagram in Fig. 3.

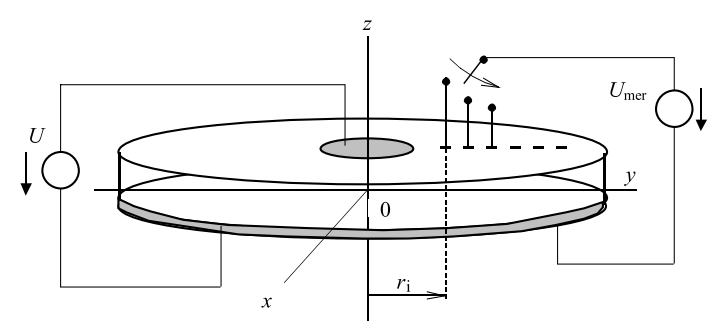

Fig. 3. Schematic diagram for the investigation of the spatial distribution of the dielectric permittivity of the material
During the measurement, a sample with a radius $R_{1}$ is positioned on the surface of conductive electrode. Another conductive electrode with a radius $R_{2}$ is placed in the axis of symmetry of the measuring system, on the upper surface of the sample. Between the electrodes, the testing voltage $U$ is applied. By means of a movable electrode with the end-point the voltage on the surface of the sample in the direction from the inner to the outer electrode was measured, with a fixed spacing between the measurement points.

The model measurement to verify the applicability of the designed method of determining the difference of the electrical potential (MEP) in examining the technological texture was carried out on alumina ceramic matrix with raw material composition: $30 \mathrm{wt} \%$ Kaolin, $15 \mathrm{wt} \%$ Clays, $30 \mathrm{wt} \%$ Feldspar and $25 \mathrm{wt} \%$ Alumina. In operational terms, the company Netzch moulding press was used to produce blanks with a diameter of $320 \mathrm{~mm}$. From the blank, a sample was cut to make the measurement.

Moisture of the pressed mass was about $21 \mathrm{wt} \% \mathrm{H}_{2} \mathrm{O}$. The temperature in the extrusion from the molding press did not exceed the value of the surface temperature of $21-22{ }^{\circ} \mathrm{C}$. The thickness of sample was $2 \mathrm{~mm}$ and applied voltage $U=1.5 \mathrm{~V}$. The voltage $U_{v}$ was measured in eight directions (I, II,...VIII) which were rotated by $45 \mathrm{deg}$. Measurements were carried out on the surface of the sample by using the scheme illustrated in Fig.3. Radii of the electrodes were $R_{1}=8,5 \mathrm{~mm}$ and $R_{2}=160 \mathrm{~mm}$. Experimental data were compared with the theoretical result (30). The obtained result is shown in Fig.4 .

\section{DISCUSSION}

The quality and efficiency of the technology of electrical insulators for very high voltage (VHV) depend on the characteristics of the used ceramic material. Many authors have dealt with the evaluation of the texture and dielectric properties of materials in the past [6-12]. In our work we scanned local changes of dielectric properties of a thin ceramic sample by means of the measurement of distribution of scalar electric potential on the sample surface. The observed discrepancies between theoretical and measured values are caused by the local inhomogeneities in the material structure. It should be noted that our theoretical result (30) was obtained for the homogeneous sample under the simplifying assumptions. However, the character of dependence (30) is significant for practical use.

In view of the observed homogeneity of the blank (untreated material) structure, the measurement results show a high degree of orientation of particles of the ceramic mass plastic components across the blank. We assume that the observed deviations in electrical potential values are an reflecting the rearranging of the texturemaking particles. The created texture and homogeneous 
environment in the volume of the blank should lead to the low reject of the produced insulators due to the presence of cracks or reducing their mechanical strength. This assumption was confirmed in technology of insulators production. The manufactured series of insulators were characterized by low levels of the rejection. The same conclusion was also obtained by method of an analysis of dimensional changes of the samples before and after firing, which were collected from the profile of the blank from the directions in which the values of the difference of the electric potential were measured.

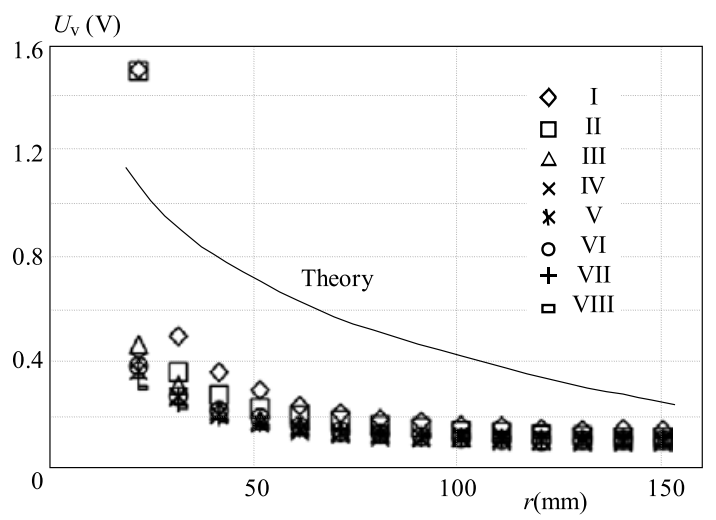

Fig. 4. Discrete symbols - data measured in different directions along diameter of the cylindrical sample. Solid line - theoretical result

\section{CONCLUSIONS}

By a model experiment of measuring the difference of the electrical potential on the surface of the cross section of blanks for the production of VHV insulators, suitability and validity of using the designed method for determining the technological texture in the blanks was demonstrated. Analysis of the obtained results confirmed the perspective of the designed method for monitoring and evaluating technological texture of materials prepared by technologies similar to production technologies of electrical porcelain - VHV insulators.

The starting point for the preparation of a model experiment was a theoretical solution of investigation of the spatial distribution of the dielectric permittivity of the material placed between two conductive electrodes (plates) with different diameters connected to the voltage $U$.

\section{Acknowledgements}

The authors thank the staff of CERAM ab for the support of research and providing samples for the experiments. This work was financially supported by grant of Slovak Republic VEGA No.1/0356/13

\section{REFERENCES}

[1] FAKhri, H.-CHEnAGHLON, A.: Phys.Lett. A 358 No. 5-6 (2006), 345-353.
[2] MEHDI, T.-DEHGHAM, M. : Phys.Scr. 72 (2005), 345-348.

[3] Fitouhi, A.-HAMZA, M. M.-BOUZEFFouR, F. : J. Approx. Theory 115 (2002), 144-166.

[4] ZHANG, J.-BELWARD, J. A. : Appl. Math\&Comp. 88 No. 2-3 (1997), 275-286.

[5] CREGG, P. J.-SVEDLindH, P.: J. Phys. A Math. Theor. 40 (2007), 14029-14031.

[6] KALUŽNÁ, M. : Influence of the texture on physical properties of ceramics for electrotechnology (Vplyv textry na fyziklne vlastnosti elektrotechnickej keramiky), FU SAV Bratislava, 1990, Thesis. (in Slovak)

[5] ŠTUBŇA, I-KOUBEK, V-KOZÍK, T. : Dynamic mechanical analysis of ceramic material (Dynamická mechanická analýza keramického materiálu), Sklář a keramik 30 No. 11 (1980), 301-303. (in Slovak)

[8] KOZÍIK, T-LABAŠ, V. : Technological Texture and its Importance in Insulators Production Technology, Proceedings of the 11th International Workshop on Applied Physics of Condensed Matter APCOM 2005, 80-85.

[9] KOZÍIK, T-LABAŠ, V-ÖLVECKÝ, Š. : A Method to determine technological texture of pressed elements (Metóda na stanovenie technologickej textúry výliskov), SILIKA No. 5-6 (2005), 131-134. (in Slovak)

[10] BOŠÁK, O.-KALUŽNÝ, J.-PRETO, J.-VACVAL, J.- KUBLIHA, M.-HRONKOVIČ, J. : Electrical properties of a rubber blend used in the tyre industry, Polymers for Advanced Technologies No. 18 (2007), 141-143.

[11] KUBLIHA, M.: Investigating structural changes and defects of non-metallic materials via electrical methods, 1-st ed., Forschungszentrum Dresden - Rossendorf, 2009.

[12] RIEDLMAJER, R.: Relationships between electrical and mechanical properties of the basalt ceramics, 1-st ed., Forschungszentrum Dresden - Rossendorf, 2009.

Received 15 April 2013

Tomáš Kozík (Prof, Ing, DrSc) graduated in physics of solid states from the Slovak University of Technology in Bratislava, Slovakia in 1969. He gained PhD in condensed matter physics and DrSc in technology of ceramic materials processing in 1989 and was appointed associate professor for physics of condensed compounds and acoustics at the Slovak University of Technology in Bratislava, later in 1994 full professor for electrical engineering materials at the Faculty of Electrical Engineering, STU in Bratislava. Since 1990 he has been working at the Department of Technology and information Technologies, University of Constantine the Philosopher in Nitra. His main research activities are focussed on physical properties and technology of the classical and progressive ceramic materials, special glasses and plastics treatment, as well as didactics of technical vocational subjects.

Stanislav Minárik (Assoc Prof) is a 49-year old University teacher and Scientist, living in Trnava, Slovakia. He has 25 years of experiences in field of Physics of Solid State. He is the author of many research articles and developed many comprehensive solutions of physical problems. He defended his PhD thesis in Institute of Physics Slovak in Academy of Science in Bratislava. The thesis was oriented on physical properties and structure of HTC superconductors based on copper oxide layers. In 2004 he was appointed associate professor at the Slovak University of Technology in Bratislava. His main research activities are focussed on theoretical problems in physics and mathematical physics. 